\title{
Investigating undergraduate students' ideas about the fate of the Universe
}

\author{
Mallory Conlon \\ University of Illinois at Urbana-Champaign, Urbana, Illinois 61801, USA \\ Kim Coble \\ San Francisco State University, San Francisco, California 94132, USA \\ Janelle M. Bailey \\ Temple University, Philadelphia, Pennsylvania 19122, USA \\ Lynn R. Cominsky \\ Sonoma State University, Rohnert Park, California 94928, USA
}

(Received 6 May 2017; published 10 November 2017)

\begin{abstract}
As astronomers further develop an understanding of the fate of the Universe, it is essential to study students' ideas on the fate of the Universe so that instructors can communicate the field's current status more effectively. In this study, we examine undergraduate students' preinstruction ideas of the fate of the Universe in ten semester-long introductory astronomy course sections (ASTRO 101) at three institutions. We also examine students' postinstruction ideas about the fate of the Universe in ASTRO 101 over five semester-long course sections at one institution. The data include precourse surveys given during the first week of instruction $(N=264)$, postinstruction exam questions $(N=59)$, and interviews. We find that, preinstruction, more than a quarter of ASTRO 101 students either do not respond or respond with "I don't know" when asked what the long-term fate of the Universe is. We also find that, though the term was not necessarily used, students tend to describe a "big chill" scenario in the preinstruction surveys, among a wide variety of other scenarios. A fraction of students describe the fate of smaller-scale systems, possibly due to confusion of the hierarchical nature of structure in the Universe. Preinstruction, students mention the Universe's expansion when describing how astronomers know the fate of the Universe but do not discuss how we know the Universe is expanding or the relationship between expansion and the fate of the Universe. Postinstruction, students' responses shift toward greater degrees of completeness and correctness.
\end{abstract}

DOI: 10.1103/PhysRevPhysEducRes.13.020128

\section{INTRODUCTION}

\section{A. Background and motivation}

While numerous astronomy education studies explore students' alternate conceptions pertaining to geocentric aspects of astronomy, particularly students' ideas on lunar phases e.g., [1] (see also Refs. [2,3], and references therein), the cause of the seasons e.g., [4-7], and the shape of Earth e.g., [8-10], only a handful of studies explore students' alternate conceptions pertaining to cosmological aspects of astronomy. These studies have focused on topics such as students' ideas about the big bang [11,12], often as only one topic among several in a broader survey of multiple areas $[13,14]$. For example, Prather et al. [11] found that large percentages of

\footnotetext{
*Corresponding author. kcoble@sfsu.edu

Published by the American Physical Society under the terms of the Creative Commons Attribution 4.0 International license. Further distribution of this work must maintain attribution to the author(s) and the published article's title, journal citation, and DOI.
}

middle school (62\%), high school (70\%), and college students (80\%) said that the big bang was an explosion of preexisting matter; similar results were found in a subsequent study [12]. Wallace et al. [12] also found that college students were generally challenged to correctly identify and interpret Hubble diagrams or galactic rotation curves, which are important pieces of evidence for the expansion of the Universe and the existence of dark matter, respectively.

Our own research team has expanded the research on cosmological topics, and the present study continues this line of inquiry. Coble et al. [15] explored students' ideas on astronomical sizes and distances and the hierarchical nature of cosmic structures, while Coble et al. [16] explored students' ideas on the composition of the Universe, including dark matter and dark energy. Trouille et al. [17] investigated students' ideas on the big bang theory and the age, expansion, and history of the Universe. Bailey et al. [18] presented the results of a nationwide, openresponse, preinstruction survey on various cosmological topics, including those discussed in Coble et al. $[15,16]$ and Trouille et al. [17]. A future analysis will explore students' ideas about the curvature of the Universe [19]. 
Our understanding of the long-term fate of the Universe is directly related to our understanding of how dark energythe mysterious force that is causing the Universe's expansion to accelerate-evolves. Dark energy makes up about $70 \%$ of the Universe's mass-energy density [20,21] and upcoming surveys will improve our understanding of the evolution of dark energy $[22,23]$. As astronomers continue to develop a deeper understanding of the Universe and its evolution, it is important that undergraduate astronomy courses effectively incorporate this new understanding into their curricula. The call for the use of modern topics in astronomy courses throughout the education community e.g., [24] and for science education reform at all levels [25-31] make it necessary to understand and develop curricula to address students' alternate conceptions on a variety of astronomy topics. However, even in the most current efforts-the Next Generation Science Standards-cosmological discussions are limited to an explanation of and the evidence for the big bang theory [32], meaning that the cutting-edge ideas of cosmological research are unlikely to be addressed at the K-12 level. Furthermore, addressing students' alternate conceptions, building on strengths, and increasing scientific literacy are imperative to teaching at all levels [25,26,29], including at the college level [33].

As seen in Deming and Hufnagel [34], most students taking an introductory astronomy course at the college level have never previously taken an astronomy course of any kind. Prior to an introductory astronomy course, students' exposure to astronomy is typically limited to middle school science curricula and, according to the National Science Board's Science and Engineering Indicators [35], information from various media outlets such as television documentaries and movies. Additionally, most of these students will not take any further astronomy classes following the introductory astronomy course [36], and the understanding these students gain from an introductory astronomy course will serve as the foundation of their astronomy knowledge. Therefore, providing introductory astronomy courses that are based on a modern view of the Universe and a modern treatment of science, which address and build on the ideas students bring to the classroom, can contribute to the development of a more scientifically literate society.

The current paper frames our research around the idea of helping move students from (potentially) thinking about cosmology in a way that does not align with that of scientists to a way that better aligns with scientific thinking. Thus, we are using a theoretical framework of conceptual change e.g., $[37,38]$. Conceptual change research assumes that students are not blank slates on which new knowledge can be written, but rather that they bring ideas that can help or hinder the learning process to new learning situations [39]. Although quite popular for many years, conceptual change research has received a number of criticisms as our understanding of cognitive science and learning processes has improved see, for example, Ref. [40]. One such criticism is that such research focuses too narrowly only on scientific content, ignoring the broader context of culture or implications of learning on students' lives [40]. Lemke argues that, "An apparent assumption of conceptual change perspectives in science education is that people can simply change their views on one topic or in one scientific domain, without the need to change anything else about their lives or their identities" (p. 301). An investigation of learning in this manner is beyond the scope of the present study, though it would be a worthy future endeavor. Despite such criticisms, we believe that identifying the ideas that students bring with them to an introductory astronomy course, which can be used as a starting point for informing instruction, as well as where students continue to have ideas different from the desired outcomes after instruction, can be valuable for both individual instructors who want to be more cognizant of their students' prior knowledge and the body of educational research more broadly. This approach also follows much of the history of research in more established topics, such as biological evolution used as an example by Lemke, whereby an investigation of content understanding and change came prior to sociocultural investigations of the topic. The present exploratory study is thus grounded in the conceptual change tradition; however, we did not use a conceptual change approach to instruction (i.e., with a specific intent to alter known nonscientific conceptions) nor are we claiming to robustly measure conceptual change such as through the use of widely used instruments (e.g., concept inventories).

\section{B. Astronomy background}

In the late 1990s, two independent teams concurrently made the first observations of the Universe's accelerated expansion. These teams, the High-z Supernova Search team led by Schmidt and the Supernova Cosmology Project led by Perlmutter, determined distances to Type Ia supernovae, which are standard candles and commonly used extragalactic distance indicators. Both teams observed highredshift, or incredibly distant, Type Ia supernovae, and obtained the same surprising results: these high-redshift Type Ia supernovae were much fainter than expected when assuming that the Universe has a constant expansion history. The explanation provided by both teams for this result was that the Universe's expansion was not constant but accelerating $[41,42]$. Though the acceleration's cause was not understood, astronomers named the cause dark energy. Schmidt and Perlmutter, along with Riess of the High-z Supernova Search, were awarded the Nobel Prize in Physics in 2011 for this discovery.

Data from all-sky surveys of the cosmic microwave background, such as WMAP [20] and Planck [21], have enabled astronomers to precisely quantify the composition of the Universe. While we know that dark energy makes up about $70 \%$ of the Universe's mass-energy density, the nature of dark energy, how it evolves, and how it might impact the Universe in the future remains uncertain. Though results 
from baryon acoustic oscillations seem to suggest that the dark energy density will remain constant (see Refs. [21,43], and references therein), new science results over the next ten years from surveys such as the Dark Energy Survey [22] and the Large Synoptic Survey Telescope [23] are intended to greatly improve our understanding of the evolution of dark energy. The Dark Energy Survey will image 5000 square degrees of the southern hemisphere's sky in an effort to describe how dark energy evolves through observations of Type Ia supernovae and weak gravitational lensing [22]. The Large Synoptic Survey Telescope will image 20000 square degrees of the southern hemisphere's sky, with one of its primary science goals focused on understanding dark energy through weak gravitational lensing observations [23]. The results from Dark Energy Survey and Large Synoptic Space Telescope should provide a more precise understanding of how dark energy, and consequently the Universe, will evolve.

The evolution of the expansion rate of the Universe is described by the Friedmann equation, which includes terms for the matter and energy density, the curvature, and the dark energy content of the Universe. Though the Friedmann equation allows for many more options, there are ultimately six sets of characteristics a Universe can have under the Big Bang model that are relevant to its fate. Each of these six sets can be observationally differentiated based on the composition of the Universe, the curvature of the Universe, and the evolution of dark energy. These six scenarios lead to three possible outcomes for the physical conditions of the Universe: a big chill (or big freeze), in which the Universe expands and cools indefinitely; a big crunch, in which the Universe eventually collapses back on itself, heating up; and a big rip, in which the Universe eventually expands so rapidly that atoms will be torn apart. The six possible characteristic sets are listed below:

(1) Closed universe: The outward pull of expansion is less than the inward gravitational pull caused by massive objects. This universe ${ }^{1}$ has positive curvature and no dark energy. This universe will eventually stop expanding and collapse back on itself. The outcome of this universe is a big crunch.

(2) Critical universe: The outward pull of expansion is exactly equal to the inward gravitational pull caused by massive objects. This universe has no curvature and no dark energy, and it will continue to expand and cool forever, though the expansion will slow down over time. The outcome of this universe is a big chill.

(3) Open universe: The outward pull of expansion is greater than the inward gravitational pull caused by massive objects. This universe has negative curvature and no dark energy, and it will continue to expand and cool forever. The outcome of this universe is a big chill.

\footnotetext{
${ }^{1}$ Here we use lowercase "universe" to mean a hypothetical universe, to distinguish it from our actual Universe.
}

(4) Constant dark energy universe: Dark energy dominates this universe's evolution, and the expansion will accelerate. This could occur for almost any amount of mass or curvature. The density of dark energy remains constant over time while that of matter decreases as the universe expands. This universe will cool down and expand forever at an increasing rate. The outcome of this universe is a big chill.

(5) Decreasing dark energy universe: Dark energy dominates this universe's evolution at the present time, and the expansion rate will accelerate for some time. However, because the density of dark energy decreases over time, eventually matter dominates the evolution of this universe. This universe then continues to expand but does so at a nonaccelerating rate. The outcome of this universe is a big chill.

(6) Increasing dark energy universe: Dark energy dominates this universe's evolution, and the expansion will accelerate. This could occur for almost any amount of mass or curvature. The density of dark energy increases over time, so this universe's expansion will accelerate so much that even molecules will be torn apart. The outcome of this universe is a big rip. Although the uncertainties about dark energy do not currently allow for a complete prediction of the fate of the Universe, three of the above listed scenarios can be eliminated based on current observations. For instance, current observations indicate that the Universe contains far too little mass to overcome the outward pull of expansion. Therefore, a closed universe (1) is ruled out by observations. Similarly, the overwhelming amount of dark energy in our Universe also indicates that we must take dark energy into account. Therefore, the critical (2) and open universes (3) do not accurately describe our Universe. We also know that the overall curvature of the Universe is zero to percent-level precision e.g., [21,44]. Therefore, sets (4), (5), and (6) are the remaining three scenarios that can describe the fate of our Universe while remaining consistent with current observations. A scenario with constant dark energy and zero curvature is most likely, pointing to an eventual outcome of a big chill for our Universe.

\section{Present study}

Our goal in this study was to examine and document the range of students' ideas regarding the long-term fate of the Universe. This study is one in a series examining the nature and frequency of students' ideas about cosmology using a mixed-methods approach, including both qualitative and quantitative data sources. The approach is similar to that used in our previous work [15-18] and is described in more detail below. Though previous studies on the Universe's expansion and dark energy probe related ideas, to our knowledge, there have been no studies specifically looking at students' ideas about the long-term fate of the Universe to date.

In Sec. II, we describe our methods, including the setting, participants, data sources, and analysis procedure. We then 
TABLE I. Undergraduate demographics for each university. ${ }^{2}$ Information about whether students had taken a prior astronomy course was collected directly on survey forms. Other demographics reported were based on each university's institutional data on the general undergraduate population.

\begin{tabular}{|c|c|c|c|c|c|c|}
\hline \multirow{3}{*}{$\begin{array}{l}\text { University } \\
\text { Chicago State University } \\
\text { (CSU) }\end{array}$} & \multirow{2}{*}{\multicolumn{3}{|c|}{ University demographics }} & \multicolumn{3}{|c|}{ Taken astronomy before? } \\
\hline & & & & \multirow{2}{*}{$\frac{\text { Yes }}{0 \%}$} & \multirow{2}{*}{$\frac{\text { No }}{65 \%}$} & \multirow{2}{*}{$\frac{\text { No response }}{35 \%}$} \\
\hline & $\begin{array}{l}78 \% \text { African American } \\
1 \% \text { Asian/Pacific Islander } \\
6 \% \text { Hispanic } \\
2 \% \text { White }\end{array}$ & $71 \%$ Female & Average age: 29 & & & \\
\hline Concord University (CU) & $\begin{array}{l}6 \% \text { African American } \\
1 \% \text { Asian/Pacific Islander } \\
1 \% \text { Hispanic } \\
87 \% \text { White }\end{array}$ & $57 \%$ Female & Average age: 23 & $6 \%$ & $60 \%$ & $34 \%$ \\
\hline $\begin{array}{l}\text { University of Nevada- } \\
\text { Las Vegas (UNLV) }\end{array}$ & $\begin{array}{l}\text { 8\% African American } \\
18 \% \text { Asian/Pacific Islander } \\
23 \% \text { Hispanic } \\
38 \% \text { White }\end{array}$ & $56 \%$ Female & Average age: 22 & $9 \%$ & $62 \%$ & $29 \%$ \\
\hline
\end{tabular}

present students' ideas on the fate of the Universe in Sec. III. In Sec. IV we discuss our most important results and in Sec. V we conclude with the implications of this work.

\section{METHODS}

\section{A. Participants and setting}

ASTRO 101 is a colloquial term for any introductory undergraduate course designed to give a broad overview of astronomy. The ASTRO 101 courses examined in this study, comprised of a convenience sample, cover the major topics typically taught in an ASTRO 101 course [45], and were taught at three American undergraduate institutions: Chicago State University (CSU), an urban minority-serving university located in the Midwest; Concord University (CU), a regional state university in Appalachia; and the University of NevadaLas Vegas (UNLV), an urban research-intensive university located in the western United States. Although individual demographic data were not collected, student demographics for the ASTRO 101 courses are generally representative of each university's undergraduate population as a whole because the courses satisfy general education requirements for all students. Table I includes details on each university's undergraduate demographics. Table I also includes information about whether students had taken astronomy prior to the course in which they were completing the survey; these data came directly from the student responses, as opposed to institutional data. Participants who responded "yes" were also asked whether this was in high school or college (typically

\footnotetext{
${ }^{2}$ Demographic citations: http://www.csu.edu/IER/documents/factBook2013-14.pdf. http://hub.concord.edu/ir/sites/hub.concord.edu.ir/files/CU\% 202013-2014\%20Common\%20Data\%20Set.pdf. https://ir.unlv.edu/IAP/Reports/Content/UndergraduateStudentProfile_ Fall2013.aspx.
}

about an even split) but no other details were asked or provided.

\section{B. Data collection}

The data set for this study included preinstruction surveys $(N=264)$ at three universities (CSU, CU, and UNLV) and postinstruction exam questions $(N=59)$ from one university (CSU). Additionally, midsemester interviews, some just before and some just after instruction on cosmology, were conducted at one university (CSU). The reported numbers were totaled over all applicable semesters unless otherwise indicated. The number of responses varied across sections for a variety of reasons, including that some students received preinstruction survey forms on other cosmological topics and some sections have lower enrollment than others. Details of the preinstruction survey questions and the postinstruction exam questions can be found in Table II.

Preinstruction surveys were given during the first week of instruction to students in ASTRO 101 classes over five semesters (Fall 2011, Spring 2012, Fall 2012, Spring 2013, and Spring 2014). The students were not prompted to use any specific terms in the preinstruction surveys; they were simply asked what they thought the long-term fate of the Universe is and how we know.

Postinstruction data from the CSU ASTRO 101 course is in the form of open-ended exam questions. The exam questions differed from the preinstruction survey questions but still probed students' ideas about the fate of the Universe. Questions on the fate of the Universe were placed on exams given during the last week of regular instruction (Fall 2010, Spring 2011, and Fall 2012) or during final exams (Spring 2013 and Spring 2014).

We should note that the preinstruction surveys were collected anonymously, and so although there is some overlap with the participants who also provided postinstruction data in the CSU subset in Fall 2012, Spring 2013, 
TABLE II. Preinstruction survey and postinstruction exam questions.

\begin{tabular}{|c|c|c|}
\hline University & Preinstruction survey questions & postinstruction exam questions \\
\hline CSU & $\begin{array}{l}\text { What is the long-term fate } \\
\text { of the Universe? How do } \\
\text { we know? } \\
(N=22) \\
\text { (Fall } 2012, \text { Spring 2013, } \\
\text { Spring 2014) }\end{array}$ & $\begin{array}{l}\text { (a) Describe two of the four }{ }^{3} \text { possible scenarios for the fate of the } \\
\text { Universe. For each case, describe what conditions will be like, } \\
\text { the curvature of the Universe, and which term (gravity, } \\
\text { expansion, or dark energy) in the Friedmann equation would } \\
\text { dominate. } \\
\text { (b) Observationally, which of the four possible scenarios } \\
\text { best describes our Universe? Explain. } \\
\text { ( } N=59) \\
\text { (Fall 2010, Spring 2011, Fall } 2012 \text {, Spring 2013, Spring 2014) }\end{array}$ \\
\hline $\mathrm{CU}$ & $\begin{array}{l}\text { What is the long-term fate } \\
\text { of the Universe? How do } \\
\text { we know? } \\
\qquad(N=35) \\
\text { (Spring 2013, Spring 2014) }\end{array}$ & $N / A$ \\
\hline UNLV & $\begin{array}{l}\text { What is the long-term fate } \\
\text { of the Universe? How do } \\
\text { we know? } \\
\qquad(N=207) \\
(5 \text { sections Fall 2011- } \\
\text { Spring 2012) }\end{array}$ & $N / A$ \\
\hline
\end{tabular}

and Spring 2014, we cannot look at changes to any individual student's ideas.

As part of our larger project on students' ideas about cosmology, semistructured interviews were conducted throughout four semesters of ASTRO 101 (Spring 2009, Spring 2010, Fall 2010, and Fall 2012) courses at CSU. Students were asked various questions about astronomical topics, including questions that probed their ideas on the fate of the Universe. Only two of these interviews were directly relevant for the present study focusing on the fate of the Universe. In Fall 2012, two students, one preinstruction and one postinstruction, were specifically asked: What is the long-term fate of the Universe? and How do we know? The student interviews are intended to provide exemplifications of students' ideas on the fate of the Universe, before and after instruction. Results from this small number of interviews should be interpreted with caution, as they may not be representative of all students in the sample or in the population of ASTRO 101 students as a whole. Therefore, we used them here for illustrative purposes only.

\section{Data analysis}

We used a mixed-methods approach, specifically a convergent parallel design [46] in collecting and analyzing the data. The use of multiple data sources can be a powerful approach to answering research questions $[47,48]$. In this case, all of the data sources were collected over multiple

\footnotetext{
${ }^{3}$ In the results, we provide more detail on why the students were taught four scenarios for the fate instead of six; in short, the three scenarios involving dark energy were collapsed into one category.
}

semesters but analyzed simultaneously. Unlike in other mixed-methods designs, such as explanatory or exploratory designs, we did not use the results from one of the data sources to inform the next.

For the preinstruction surveys, we carried out a content analysis [49] through an iterative process of open coding to identify themes that emerged from the set of responses. One researcher read the students' responses to each survey question and recorded the common themes seen in the responses. This process was repeated until no new themes emerged and the resulting list was considered comprehensive. Each theme was then assigned a short code and codes were grouped where appropriate. All responses $(N=264)$ were coded for themes. The themes were then quantified by looking at frequency of their appearance across the participating schools and as a whole.

We defined an emergent theme as a response type that was coded at least 3 times throughout the students' preinstruction responses. Codes that appeared fewer than 3 times throughout the students' preinstruction responses were coded as other. We then identified the fraction of students who discussed a given theme in their response. Thematic codes and examples of each code for the preinstruction surveys can be found in Tables III and IV. It should be noted that, in most cases, the students' responses are limited to what is presented here-in other words, these phrases are not segments of a response but rather the whole response.

Preinstruction surveys and postinstruction exam essays were also coded for degree of completeness and correctness using the rubric in Table $\mathrm{V}$ and comparing students' responses to a desired response (which may contain more than one element). We chose to code for degree of 
TABLE III. Thematic coding scheme for the preinstruction survey question, "What is the fate of the Universe?".

\begin{tabular}{ll}
\hline \hline Thematic code & Example \\
\hline I don't know or no response & "Not sure, I have no clue" \\
Big chill & "Everything expands out until everything is inactive" \\
We don't know & "We don't know" \\
Big crunch & "To collapse in on itself" \\
Universe will end & "At some point it will terminate." \\
Objects will die & "The sun will explode, killing all life in our Universe" \\
Universe gets sucked into a black hole or becomes & "All stars will burn out and all matter will be sucked into \\
a black hole & black holes." \\
Things die and are reborn & "Stars will die and get reborn" \\
Universe will blow up or explode & "It will eventually blow up." \\
Universe will be destroyed & "It will go on for eternity." \\
Universe will never end & "We do not know fully...no way to tell overall." \\
We'll never know & "That galaxies will merge together" \\
Galaxies or universe will merge & "I've heard the fabric of space will tear due to the \\
Big rip & Universe constantly expanding." \\
Universe is always changing & "The Universe is constantly changing" \\
Universe will live billions of years & "It took billions of years to form so I would assume the \\
lifespan is billions of years." & "Only God knows" \\
Nonscientific & "Eventually will fill up" \\
Other &
\end{tabular}

TABLE IV. Thematic coding scheme for the preinstruction survey question, "How do we know the fate of the Universe?".

\begin{tabular}{ll}
\hline \hline Thematic code & Example \\
\hline I don't know or No response & "I don't know how we know" \\
We don't know & "We don't know" \\
Universe is expanding & "We know by the scientific research that has been conducted." \\
Know from research or science/theory & "[By] looking at other galaxies and what's happening in space" \\
Make observations & "We know this because stars die at some point and the sun is a star." \\
Stellar evolution & "I feel there is no way to know or predict it." \\
There is no way to know & "Heard it somewhere" \\
Authority figure or heard before & "Evidence shows that the Universe is getting stretched by Dark Energy" \\
Dark energy & "I read the bible" \\
Nonscientific & "Everything ends" \\
Other &
\end{tabular}

TABLE V. General rubric for correctness.

\begin{tabular}{|c|c|}
\hline Code & Description \\
\hline Correct $(\mathrm{C})$ & The response was complete and contained no incorrect statements. \\
\hline Incomplete (I) & $\begin{array}{l}\text { The response was missing one or more of the identified elements required for a } \\
\text { correct response. The response contained no incorrect statements. }\end{array}$ \\
\hline Partial (P) & The response contained both incorrect and correct statements. \\
\hline Wrong $(\mathrm{W})$ & No statements in the response matched the identified elements of a correct response. \\
\hline True but irrelevant $(\mathrm{T})$ & $\begin{array}{l}\text { The included statements were true but did not address the question in any } \\
\text { meaningful way. }\end{array}$ \\
\hline Nonscientific (NS) & Nonscientific response \\
\hline No response (NR) & No response \\
\hline
\end{tabular}


correctness in addition to themes in order to more easily compare responses across institutions and before and after instruction at a single institution. We will provide examples of responses to specific questions and their codes along with the results in Sec. III.

\section{Validity}

The validity of the survey coding and interpretation was established primarily through the use of a multimember research team, including the authors of this article. Coding by two members of the author team ensured a common understanding of the responses as well as our interpretations of them. Other members of the project served as peer debriefers [50] who reviewed aspects of the study at various points in time and contributed to the credibility of the interpretations.

Interrater reliability, as calculated by kappa, ranged from 0.618 to 0.809 (for all, $p<0.001$ ), depending upon the question and coding scheme (i.e., thematic codes or correctness codes); these values of kappa are considered "substantial" or better (Landis and Koch, as cited in Ref. [51], p. 124). For those responses on which the codes differed between researchers, the selections were negotiated as needed to come to $100 \%$ agreement on the final codes to be assigned. Codes were recorded for each question and documented in a spreadsheet. The final negotiated codes were used for the results presented below.

We used the Kruskal-Wallis (KW) test to determine whether we could aggregate results from different course sections. The KW test is a nonparametric method for testing the hypothesis that three or more sample populations (in our case, the sample populations are the coded student responses from each semester) have the same mean distribution, against the hypothesis that they differ [52]. One advantage of the KW test is that it can be applied to data sets in which the number of values from each semester are of equal or unequal lengths. That is, the KW test is valid for comparing responses from different numbers of students across semesters. To use the KW test, each sample population must have 5 or more student responses.

For this study, we used a significance level of 0.05 . If our $p$ value was greater than 0.05 , we did not reject the null hypothesis that the semester results come from the same parent population. In other words, $p>0.05$ meant we could aggregate our sample population, because the sample populations did not appear to differ significantly from one another. For the ASTRO 101 preinstruction surveys and the CSU postinstruction exam questions, we ran KW tests to determine whether the different semesters' data could be combined. The $p$ value was greater than 0.05 in every case, so we are confident in aggregating results across semesters and universities.

\section{RESULTS}

In this section, we present ASTRO 101 preinstruction results from all participating institutions, first by thematic coding and then based on the rubric for degree of completeness and correctness. We then consider pre and postinstruction results, compared by correctness, from CSU alone. Some description of the curriculum may be given as context for the environment in which the development of the students' ideas has taken place; however, it is not our intention to provide detail in this regard or to measure the effectiveness of the curricula.

\section{A. ASTRO 101 preinstruction results: Thematic coding}

Here we present our thematic results on students' ideas of the fate of the Universe and how we know the fate of the Universe. The response plots for each question are organized from top to bottom from highest to lowest frequency of response, except for "nonscientific" and "other," which are always listed at the bottom. As stated in Sec. II, individual student responses could be coded under multiple themes.

After thematically coding responses to "What is the fate of the Universe?" for the preinstruction surveys, we found that the most common response from ASTRO 101 students, accounting for $28 \%$ of responses, was either I don't know or no response. The remaining responses were spread out amongst 17 thematic codes. Of these responses, the most common response was a big chill scenario (22\%), followed by we don't know (17\%), and a big crunch scenario (15\%). Though not a common response, we found that $7 \%$ of responding ASTRO 101 students mentioned either the fate of the planets, Earth, Sun, or Solar System in place of the fate of the Universe. Full results can be found in Fig. 1.

In the ASTRO 101 preinstruction surveys, students were also asked "How do we know the fate of the Universe?" We coded 49\% of ASTRO 101 responses as I don't know/no response, which was the most common response. Of the remaining responses, the most popular was we don't know (23\%). In the next most popular response, students noted that the Universe is currently expanding but did not discuss how expansion relates to or affects the fate of the Universe (17\%). A simple statement such as "the Universe is expanding" describes current observations of the Universe and does not give any description of the Universe in the future. This response differs from the students' responses that were coded as a big chill response to "What is the fate of the Universe?" which is marked by statements such as the Universe will expand forever. Again, though not the most common response, 10\% students used the fate of smaller objects, such as the Sun and stars, as evidence of how we know the fate of the Universe. The full results can be found in Fig. 2.

\section{B. ASTRO 101 preinstruction results: Correctness}

Responses from all ASTRO 101 students were also coded for correctness and completeness based on responses to both parts of the preinstruction survey. Table VI shows examples of responses of varying degrees of completeness and correctness for the preinstruction surveys. 


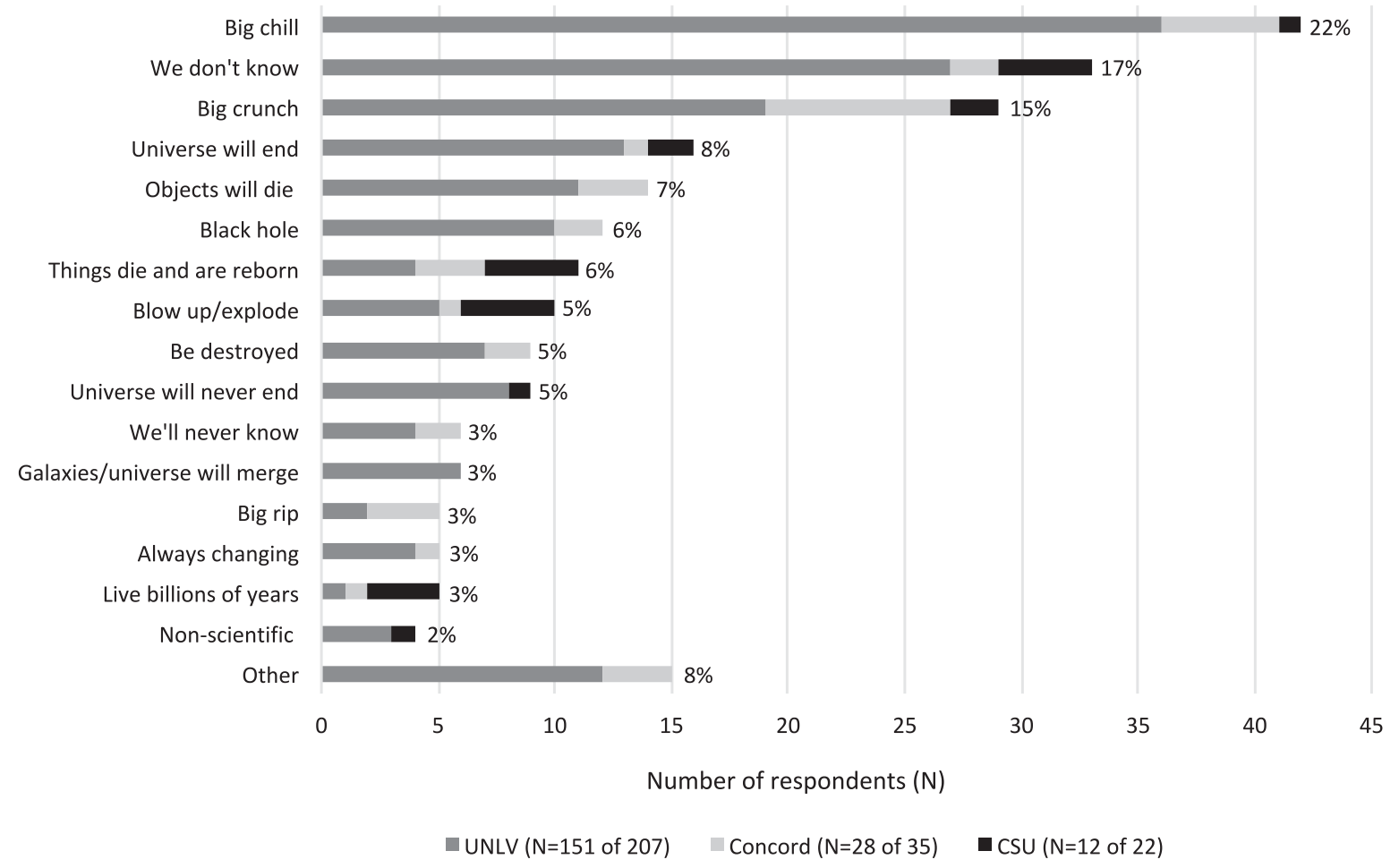

FIG. 1. ASTRO 101 preinstruction survey responses, thematic coding-What is the fate of the Universe? The horizontal axis is the number of responses coded under each theme ( $N=191$ of 264 students responded to this question). The percentage for each theme (out of the total number of students responding) is listed at the end of each category. The percentages may add up to more than $100 \%$ because each response may be coded with more than one theme.

Response profiles from different types of institutions are similar (Fig. 3). In order of correctness, we found that overall $1 \%$ of responses were coded as correct, $16 \%$ were coded as incomplete, $11 \%$ were coded as partial, $43 \%$ were coded as wrong, and $24 \%$ were coded as no response. Responses of I don't know were coded as no response, whereas responses that supplemented I don't know with another idea could be coded as incomplete, partial, or

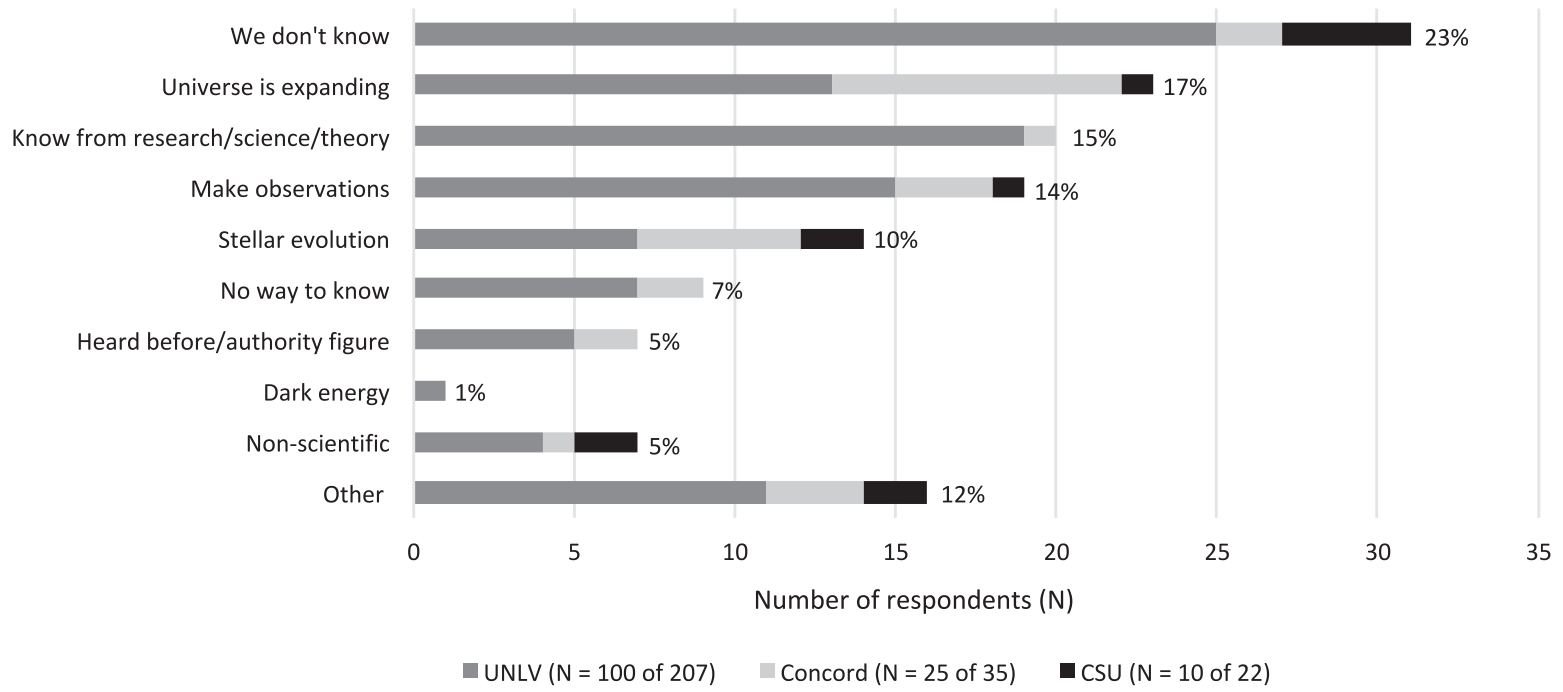

FIG. 2. ASTRO 101 preinstruction survey responses, thematic coding — How do we know the fate of the Universe? The horizontal axis is the number of responses coded under each theme ( $N=135$ of 264 students responded). The percentage for each theme (out of the total number of students responding) is listed at the end of each category. The percentages may add up to more than $100 \%$ because each response may be coded with more than one theme. 
TABLE VI. Examples of responses to preinstruction surveys, coded for degree of correctness.

Question: What is the long-term fate of the universe? How do we know?

Desired response: The universe will continue to expand increasingly quickly because of dark energy. The Universe ends in a "big chill" or "big rip." We know from observations of the CMB and a Hubble diagram (redshift measurements) of Type Ia supernovae."

Code Example

Correct (C)

Incomplete (I)

Partial (P)

Wrong (W)

True but irrelevant $(\mathrm{T})$

Nonscientific (NS)

No response (NR)
"To continue expanding. Because of redshift measurements that have been studied."

"To keep expanding."

"Increasingly rapid expansion into an eventual collapse. Models utilizing our understanding of physics and the observable characteristic of the universe."

"It is expanding because stars are separating more and more from each other."

"It will eventually blow up. I think it's because we have seen many stars erupt, so the universe will follow suit."

"That galaxies will merge together."

"We do not know, only God knows"

"I don't know" or N/A wrong. Responses of we don't know were coded as incomplete, while those that supplemented we don't know with another thematic code were coded as either incomplete or partial. This differs from how we don't know responses were treated in Coble et al. [15,16] and Trouille et al. [17], because we don't know is a more reasonable response given the current understanding of dark energy among astronomers. We don't know responses differ from we'll never know responses, which were coded as wrong.

\section{CSU ASTRO 101: Comparison of pre and postinstruction results}

ASTRO 101 students at CSU were asked a question pertaining to the fate of the Universe on an exam, either in the last week of classes or during finals week. The students had one lecture and one homework assignment on the fate of the Universe prior to taking the exams, where the question on the exams was similar to a question asked about the fate of the Universe on a homework assignment.
The students were taught four scenarios for the fate of the Universe. The three scenarios involving dark energy described in Sec. I were collapsed into a single category, accelerating universe, both in lecture and in their textbook. As our understanding of dark energy and its effect on the expansion of the Universe becomes better understood, we will be able to observationally distinguish whether dark energy is constant, increasing, or decreasing, as reflected in the six-category scheme described in the Introduction. The four scenarios described to students were the following:

(1) Closed universe: The outward pull of expansion is less than the inward gravitational pull caused by massive objects. This universe has positive curvature and no dark energy. This universe will eventually stop expanding and collapse in on itself. The fate of this universe is a big crunch.

(2) Critical universe: The outward pull of expansion is exactly equal to the inward gravitational pull caused

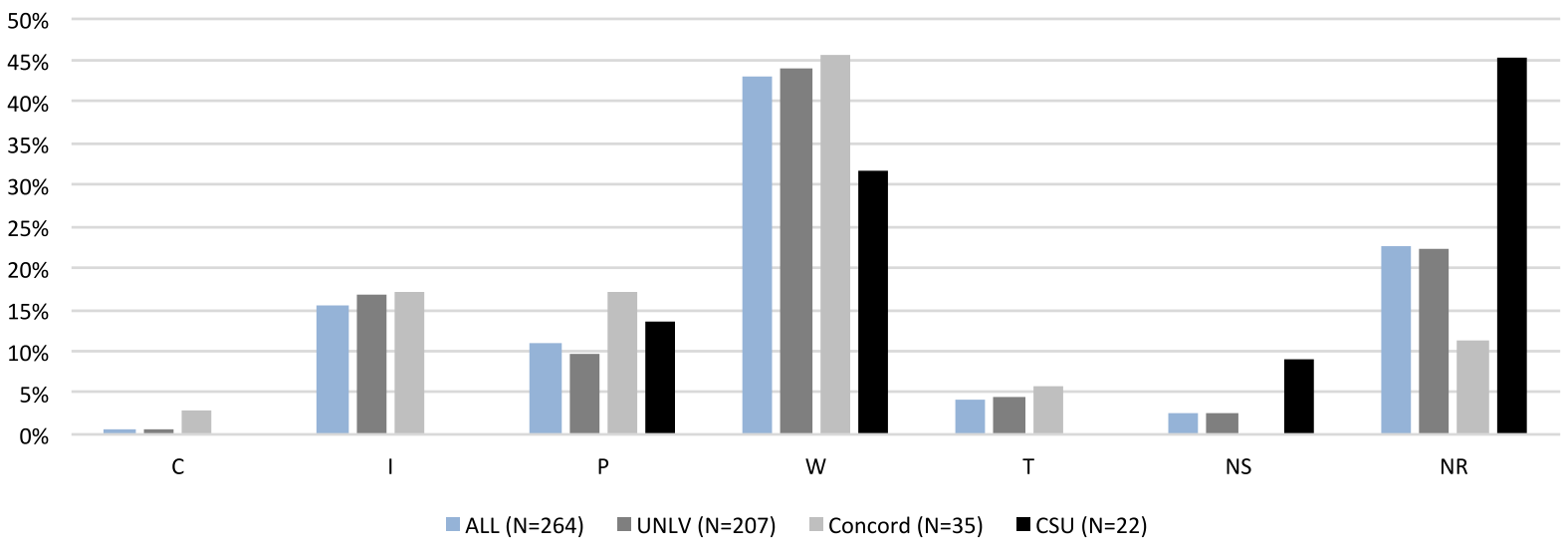

FIG. 3. ASTRO 101 preinstruction survey responses, coded for degree of correctness for both the fate and how we know combined $(N=264)$. 
TABLE VII. Examples of responses to postinstruction CSU exam question part (a), coded for degree of correctness.

Question: Describe two of the four possible scenarios for the fate of the Universe. For each case, describe what conditions will be like, the curvature of the Universe, and which term (gravity, expansion, or dark energy) in the Friedmann equation would dominate. Desired response: Any two of the following:

- Gravity term dominates, positive curvature, Universe eventually stops expanding and collapses, big crunch.

- Gravity balances expansion, no curvature, Universe expands forever increasingly slowly, big chill.

- Expansion term dominates, negative curvature, Universe expands forever, big chill.

- Dark energy dominates, Universe can have any curvature, Universe expands increasingly quickly, big chill or big rip.

\begin{tabular}{ll}
\hline Code & Examples \\
\hline Correct (C) & "1. Universe go on forever as is, but more and more slowly. Expansion balanced by gravity. Flat \\
& geometry.; 2. Universe would have saddle geometry and expansion would slow down.; 3. Universe \\
collapse: big crunch, gravity wins.; 4. Universe accelerating- dark energy." & "1. The universe could collapse.; 2. The universe will continue to expand." \\
Incomplete (I) & "Collapse-(gravity) would dominate, flat, cold.; Expand-(expansion) would dominate, flat, cold." \\
Partial (P) & "1. Exploding: extreme hot and hard to breathe, and dark energy would be the term use in which dominate \\
Wrong (W) & Friedmann equation." \\
\hline \hline
\end{tabular}

TABLE VIII. Examples of responses to postinstruction CSU exam question part (b), coded for degree of correctness.

Question: Observationally, which of the four possible scenarios best describes our Universe? Explain.

Desired response: Observationally, we know that the Universe contains dark energy and has no observable curvature overall. It will continue to expand increasingly faster. We also know this from CMB observations and a Hubble diagram of Type Ia supernovae.

Code Examples

Correct $(\mathrm{C})$

Incomplete (I)

Partial (P)

Wrong (W)

True but irrelevant $(\mathrm{T})$
"No (zero) curvature describes the universe better because the CMB proved that the universe will keep expanding forever on."

"Expansion because our universe is steadily expanding further, further away."

"Our universe is expanding faster but not too fast so that it is coasting, allowing gravity and expansion to be equal."

"I think the expansion will only go for so long, then it will behind to contract because of gravity, I don't think it makes sense to expand forever I think its gonna reach a maximum, then gravity will pull the universe back in."

" $74 \%$ hydrogen, $24 \%$ helium $2 \%$ other or $4 \%$ regular matter, $23 \%$ exotic matter, $73 \%$ dark energy" by massive objects. This universe has no curvature and no dark energy, and it will continue to expand and cool forever, though the expansion will slow down over time. The fate of this universe is a big chill.

(3) Open universe: The outward pull of expansion is greater than the inward gravitational pull caused by massive objects. This universe has negative curvature and no dark energy, and it will continue to expand and cool forever. The fate of this universe is a big chill.

(4) Accelerating universe: Dark energy dominates this universe's evolution. The universe's expansion will accelerate, causing the universe to expand and cool forever. This could occur for almost any amount of mass or curvature. The fate of this universe is a big chill or a big rip.

As mentioned in Table II, the postinstruction exam question on fate of the Universe had two parts. In part (a), students were asked to describe two of the four possible fates of the Universe learned in class and were prompted to use words such as curvature, gravity, expansion, and dark energy. In part (b), students were asked to observationally defend the most plausible fate of the Universe. Tables VII and VIII give examples of responses to the exam question for parts (a) and (b), respectively.

Exam responses were coded for correctness and completeness and were compared with preinstruction responses from CSU (Fig. 4). We see a shift from preinstruction responses being dominated (in percentages) by wrong and no-response to postinstruction responses being dominated (again, in percentages) by partial and incomplete. Note that this does not say anything about the number of students who might directly shift from one code to another, as such an analysis is not possible with our data.

\section{DISCUSSION}

\section{A. Discussion of results}

In this study, we have thus far described our data set of students' ideas about the long-term fate of the Universe. We 


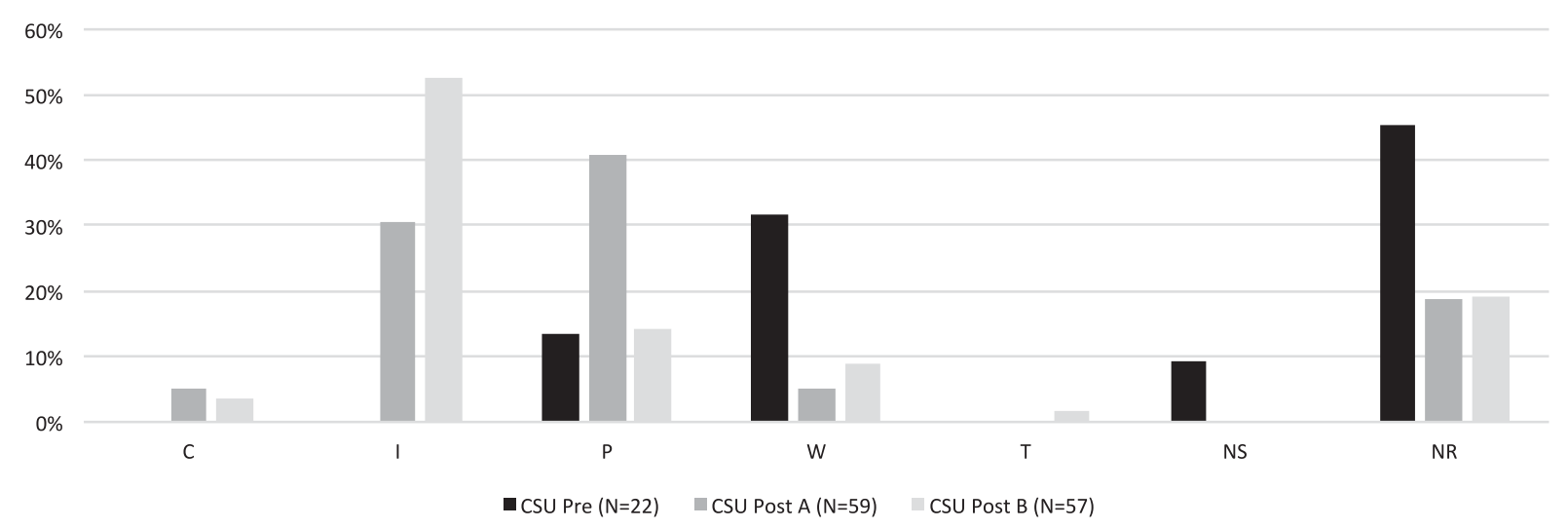

FIG. 4. CSU ASTRO 101 postinstruction exam question parts (a) $(N=59)$ and (b) $(N=57),{ }^{4}$ coded for correctness, in comparison with CSU preinstruction results $(N=22)$, also coded for correctness.

used multiple data sources, including preinstruction surveys and postinstruction exam responses, to probe students' ideas on the fate of the Universe. Here we will discuss our major findings for ASTRO 101 students at all institutions preinstruction and ASTRO 101 students at CSU pre and postinstruction. We use interviews where we asked the questions, What is the fate of the Universe and How do we know, to illustrate some of the students' thought processes.

We found that ASTRO 101 students generally come into their respective courses with a wide variety of ideas on the fate of the Universe, despite a lack of coverage of this material in K-12 science education. The large number of commonly occurring, or emergent, thematic codes suggests this to be the case. For instance, responses in our sample had 17 emergent codes at the time of the preinstruction survey (other than I don't know or no response). Students at all of the institutions surveyed entered ASTRO 101 with a similarly large number of ideas and level of correctness about the long-term fate of the Universe.

One student's response in a preinstruction interview illustrates common themes seen in the preinstruction surveys, such as a big chill scenario and that we know the fate from observations. When asked about the longterm fate of the Universe, at first the student simply replied,

"I think it will go on forever."

After prompting, and being asked how astronomers know, the student further elaborated,

"Well, the Universe is expanding and I think that just based on the amount [of] size of the Universe that we at least know of and that...is just based off the light that we're able to see from the farthest points that it

\footnotetext{
${ }^{4}$ There were 59 students who completed both parts A and B on the exam, however, 2 of the responses on part B were unreadable. These responses were eliminated from analysis and so the percentages for part B are out of 57 .
}

probably.... That it is just so large that it probably goes on forever [in time] and it probably always will."

and described the observations,

"By knowing the speed of light, knowing the... colors of the spectrum of different objects."

In the preinstruction surveys, a small subset of our sample of ASTRO 101 students discussed the fate of Earth, other planets, the Sun, or our Galaxy (small-scale fate) instead of the fate of the Universe (large-scale fate). As seen in Fig. 1, more than 7\% of ASTRO 101 students who responded discussed the small-scale fate of the Universe in place of the large-scale fate and $10 \%$ described the fate of Sun and other stars as reasoning for how we know. As seen in Refs. [11,12,15], students generally have difficulty describing the hierarchical nature of structure in the Universe prior to instruction. For example, a correct description of the structure within the Universe would be that solar systems are in galaxies, and galaxies are in the Universe. Coble et al. [15] report that only $18 \%$ of students were able to correctly describe the hierarchical structure of the Universe preinstruction.

This difficulty grasping the hierarchical structure of objects in the Universe coupled with the results from the present study (e.g., the results in Fig. 1) suggests possible conflation between the fate of the planets, Sun, or galaxies, with the fate of the Universe. A deeper probe of students' ideas, such as preinstruction interviews that discuss both small and large-scale fates, is needed to differentiate conflation of small and large-scale fates from a misunderstanding of the question or some other interpretation.

When asked prior to instruction how we know the fate of the Universe, nearly half of the students did not respond. Of those who responded to the question, the most popular response (23\%) was we don't know. The next most popular 
response (17\%) used the Universe's current expansion to describe how astronomers know the Universe's fate. Instead of describing the future of the Universe by saying "the Universe will continue to expand," students simply say, for example, "the Universe is expanding".

Students in the CSU ASTRO 101 sections appear to better grasp the idea of the fate of the Universe by the end of their courses. More students responded to the question postinstruction than preinstruction (i.e., there were fewer responses coded as no response), although that may be due to the exam format. Because the postinstruction data varied in structure from the preinstruction data, we cannot make a direct comparison, but responses moved in the direction of greater completeness and correctness from pre to postinstruction, with a majority of responses coded as incomplete or partial instead of no response or wrong.

A postinstruction interview with a second student, who correctly described a big rip and big chill scenario on a postinstruction exam, hints at a disbelief of recent scientific findings despite being able to describe them:

"The issue with that question is that would suggest that there actually is a long-term fate of the Universe ... I believe that and I only believe this because dark energy hasn't been studied in great detail to refute this just yet. I believe that gravity will eventually slow down the speed of inflation in the Universe and everything will eventually come back in on itself, a big crunch essentially".

The student continues on to describe the current scientific consensus:

"Now everything that has been proven thus far says that no, dark energy will take over".

And elaborates that he thinks differently from that consensus because,

“... there are so many other things that could possibly be... knowing the unpredictably of the universe and all of its various ways, I just feel like there is nothing to say that it may not be the case that expansion may slow to a point where gravity wins".

The student interprets a graph of recent results showing that the expansion rate was slower in the past than it is today as evidence that something different might happen in the future:

"there was indeed a time at which the universe was expanding slower and from the actual graph that I saw, the curve seemed to have an end point that may or may not suggest that this is sort of [a] cycle... this obviously goes against what everybody else believes, what all the scientists believe because they believe that the universe is expanding faster and faster. Well I believe that based upon that graph of newly discovered data that this is a continual, like a sine wave of continual speeding and slowing”.

Here the student touches on themes of things dying and being reborn, seen in the preinstruction surveys. The interview also hints at the resilience of students' mental models and the idea that we don't know what the Universe will do because it is an unpredictable place of many possibilities.

The results of this study are also related to the findings from Trouille et al. [17] on the expansion of the Universe and Coble et al. [16] on dark energy. As reported in those papers, in preinstruction interviews in which students were asked how the Universe has changed over time, two out of three students discussed the expansion. In nine postinstruction interviews, all students described the expansion and two stated that the expansion was accelerating. A third of the students referred to the usefulness of a class demonstration on the expansion of the Universe. The demonstration featured a stretchy rubber physical therapy band with pictures of galaxies stapled to it, in which the band itself (representing space) can expand but the individual galaxies do not. Prior to instruction, students have little understanding of dark energy. While they can learn about how much of the Universe is believed to be dark energy and its impact (accelerated expansion), their understanding is fairly superficial, lacking in detail or nuance.

\section{B. Limitations of the study}

This exploratory study provides a starting point for understanding students' ideas about the fate of the Universe. One of the challenges involves the lack of detailed responses to questions at both pre and postinstruction. In the preinstruction surveys, students typically responded with only a few words or a single sentence, limiting our ability to probe their understanding in depth. There is also some limitation in using exam data to evaluate postinstruction understanding. Although students might have written more on open-ended exam questions, the high-stakes nature of this setting (particularly compared to the anonymous preinstruction surveys) may impact the responses in a way that is difficult to predict here. For example, a student may answer a question the way they learned as correct during class while maintaining a belief that does not align with scientific consensus. Additionally, the differences between wording of the questions at pre and postinstruction and the anonymous collection at preinstruction, means that direct comparisons of individual learning are not possible.

Another limitation of this study is the interviews. Overlap existed between interviews within the larger project, but the semistructured nature of them also meant that some topics were addressed in only a small number of interviews. This was the case with the fate of the Universe, 
which was only directly asked in two interviews, thus limiting our ability to dig deeper into students' ideas.

These limitations within the data collection naturally restrict the inferences that can be made as a result of this study. It is intended to provide a starting point on which additional studies could be launched. For example, as noted in Sec. I, although this study is grounded in conceptual change, we did not design any particular instructional intervention to attempt to move students toward greater scientific understanding. Such an endeavor is a natural next step that could be informed by our results.

\section{IMPLICATIONS AND CONCLUSIONS}

Astronomers still do not fully understand the nature of dark energy, in particular its future evolution. Dark energy is a substantial component of our Universe, and, as a consequence, astronomers cannot precisely predict the fate of the Universe without knowing dark energy's effect on the Universe in the future. As our understanding of how dark energy evolves with results from ongoing and upcoming astronomical surveys, so will our understanding of the ultimate fate of the Universe.

Teaching about the fate of the Universe in an introductory astronomy course provides a unique opportunity to expose students to the tentative nature of science, which is a major component of Project 2061's Benchmarks for Science Literacy [26]. In order to achieve this, it may not be sufficient for students to hear that there are different possibilities for the fate of the Universe. Rather, if they are also taught about how such different possibilities would be evaluated within the scientific community, they could better understand both the possible fates and how science works.

Through the analysis of preinstruction surveys, postinstruction exam questions, and interviews, we have identified common ideas students bring to the classroom about the fate of the Universe. Similar responses are seen across instrument types and institution types, including the following:

- Two of the most popular themes that emerged, big chill and big crunch, are possible fates that are discussed by cosmologists; these ideas might inform classroom instruction.
- Similarly, the popular response we don't know might be explicitly addressed in a discussion of scientific questions where we have some data but which are not yet settled.

- The known conflation of Universe with smaller systems within the Universe, such as the Solar System, may lead to confusion about the fate of such smallscale systems with the fate of the Universe as a whole.

- While many preinstructional responses are coded as wrong or a nonresponse, after some instruction, responses shift in a positive direction (toward incomplete or partially correct).

It has been known for decades that students are not "blank slates" when it comes to instruction $[39,53]$. This study provides insights into what ideas students bring with them to an ASTRO 101 course, so that instructors might be aware and ideally build upon those ideas that can create scaffolding toward scientific understanding. The identification of these ideas further provides a starting point for researchers who would better understand conceptual change on this topic or perhaps take on a sociocultural approach [40] to considering how students conceive of the fate of the Universe. For example, future work in this area might include a larger qualitative study, with detailed interviews that occur when students are in the process (i.e., both pre and postinstruction) of learning what we know about the fate of the Universe, how we know it, and how scientists incorporate new information in a field under active study.

\section{ACKNOWLEDGMENTS}

This work was supported by NASA Grant No. NNX10AC89G and by the Education and Public Outreach program for NASA's Fermi Gamma-ray Space Telescope, the Illinois Space Grant Consortium, and NSF CCLI Grant No. 0632563 at CSU. This work would not have been possible without the participation of students from CSU, CU, and UNLV. Also, the first author wants to give special thanks to Brian Fields for valuable discussions that shaped this paper.
[1] R. S. Lindell, Ph.D. thesis, The University of NebraskaLincoln, 2001.

[2] J. M. Bailey and T.F. Slater, A review of astronomy education research, Astron. Educ. Rev. 2, 20 (2003).

[3] A. Lelliott and M. Rollnick, Big ideas: A review of astronomy education research 1974-2008, Int. J. Sci. Educ. 32, 1771 (2010).
[4] R. K. Atwood and V. A. Atwood, Preservice elementary teachers' conceptions of the causes of seasons, J. Res. Sci. Teach. 33, 553 (1996).

[5] E. Kikas, Teachers' conceptions and misconceptions concerning three natural phenomena, J. Res. Sci. Teach. 41, 432 (2004).

[6] M. Zeilik and V. J. Morris, An examination of misconceptions in an astronomy course for science, mathematics, 
and engineering majors, Astron. Educ. Rev. 2, 101 (2003).

[7] J. Ojala, Lost in space? The concepts of planetary phenomena held by trainee primary school teachers, Int. Res. Geograph. Env. Educ. 6, 183 (1997).

[8] S. Vosniadou and W. F. Brewer, Mental models of the Earth: A study of conceptual change in childhood, Cogn. Psychol. 24, 535 (1992).

[9] S. Vosniadou and W. F. Brewer, Mental models of the day/night cycle, Cogn. Sci. 18, 123 (1994).

[10] S. Vosniadou, C. Ioannides, A. Dimitrakopoulou, and E. Papademetriou, Designing learning environments to promote conceptual change in science, Learn. Instr. 11, 381 (2001).

[11] E. E. Prather, T. F. Slater, and E. G. Offerdahl, Hints of a fundamental misconception in cosmology, Astron. Educ. Rev. 1, 28 (2002).

[12] C. S. Wallace, E. E. Prather, and D. K. Duncan, A study of general education astronomy students' understandings of cosmology. Part IV. Common difficulties students experience with cosmology, Astron. Educ. Rev. 11, 010104 (2012).

[13] H. Kalkan and K. Kiroglu, Science and nonscience students' ideas about basic astronomy concepts in preservice training for elementary school teachers, Astron. Educ. Rev. 6, 15 (2007).

[14] R. Trumper, University students' conceptions of basic astronomy concepts, Phys. Educ. 35, 9 (2000).

[15] K. Coble, C. T. Camarillo, L. E. Trouille, J. M. Bailey, G. L. Cochran, M. D. Nickerson, and L. R. Cominsky, Investigating student ideas about cosmology I: Distances and structure, Astron. Educ. Rev. 12, 010102 (2013).

[16] K. Coble, M. D. Nickerson, J. M. Bailey, L. E. Trouille, G. L. Cochran, C. T. Camarillo, and L. R. Cominsky, Investigating student ideas about cosmology II: Composition of the universe, Astron. Educ. Rev. 12, 010111 (2013).

[17] L. E. Trouille, K. Coble, G. L. Cochran, J. M. Bailey, C. T. Camarillo, M. D. Nickerson, and L. R. Cominsky, Investigating student ideas about cosmology III: Big Bang theory, expansion, age, and history of the universe, Astron. Educ. Rev. 12, 010110 (2013).

[18] J. M. Bailey, K. Coble, G. L. Cochran, D. M. Larrieu, R. Sanchez, and L.R. Cominsky, A multi-institutional investigation of students' preinstructional ideas about cosmology, Astron. Educ. Rev. 11, 010302 (2012).

[19] K. Coble, M. Conlon, and J. M. Bailey, Investigating undergraduate students' ideas about the curvature of the universe, Phys. Rev. Phys. Educ. Res. (to be published).

[20] G. Hinshaw et al., Nine-year Wilkinson Microwave Anisotropy Probe (WMAP) observations: Cosmological parameter results, Astrophys. J. Suppl. Ser. 208, 19 (2013).

[21] Planck Collaboration, Planck 2015 results. XIII. Cosmological parameters, Astron. Astrophys. 594, A13 (2016).

[22] Dark Energy Survey Collaboration, The dark energy survey, arXiv:astro-ph/0510346.

[23] Z. Ivezic et al., LSST: From science drivers to reference design and anticipated data products, arXiv:0805.2366.

[24] J. M. Pasachoff, What should college students learn? Phases and seasons? Is less more or is less less?, Astron. Educ. Rev. 1, 124 (2002).
[25] American Association for the Advancement of Science, Science for All Americans (Oxford University Press, New York, 1990).

[26] American Association for the Advancement of Science, Benchmarks for Science Literacy (Oxford University Press, New York, 1993).

[27] M. A. Fox and N. Hackerman, Evaluating and Improving Undergraduate Teaching in Science, Technology, Engineering, and Mathematics (National Academies Press, Washington, DC, 2003).

[28] J. D. Bransford, A. L. Brown, and R. R. Cocking, How People Learn: Brain, Mind, Experience, and School (expanded edition) (National Academy of Sciences, Washington, DC, 2000), p. 342.

[29] National Research Council, National Science Education Standards (National Academies Press, Washington, DC, 1996).

[30] National Research Council, A Framework for K-12 Science Education: Practices, Crosscutting Concepts, and Core Ideas (National Academies Press, Washington, DC, 2012).

[31] National Research Council, Learning and Understanding: Improving Advanced Study of Mathematics and Science in U.S. High Schools (National Academies Press, Washington, DC, 2003).

[32] NGSS Lead States, Next Generation Science Standards: For States, by States (The National Academies Press, Washington, DC, 2013).

[33] M. S. Donovan and J. D. Bransford, How Students Learn: Science in the Classroom (The National Academies Press, Washington, DC, 2005).

[34] G. L. Deming and B. Hufnagel, Who's taking ASTRO 101?, Phys. Teach. 39, 368 (2001).

[35] National Science Board, Report No. NSB-02-1, Arlington, VA, 2002.

[36] A. L. Rudolph, E. E. Prather, G. Brissenden, D. Consiglio, and V. Gonzaga, A national study assessing the teaching and learning of introductory astronomy part II: The connection between student demographics and learning, Astron. Educ. Rev. 9, 010107 (2010).

[37] J. A. Dole and G. M. Sinatra, Reconceptalizing change in the cognitive construction of knowledge, Educ. Psychol. 33, 109 (1998).

[38] G. J. Posner, K. A. Strike, P. W. Hewson, and W. A. Gertzog, Accommodation of a scientific conception: Toward a theory of conceptual change, Sci. Educ. 66, 211 (1982).

[39] A. E. Lawson, The acquisition of biological knowledge during childhood: Cognitive conflict or tabula rasa?, J. Res. Sci. Teach. 25, 185 (1988).

[40] J. L. Lemke, Articulating communities: Sociocultural perspectives on science education, J. Res. Sci. Teach. 38, 296 (2001).

[41] S. Perlmutter et al., Discovery of a supernova explosion at half the age of the universe, Nature (London) 391, 51 (1998).

[42] A. G. Riess et al., Observational evidence from supernovae for an accelerating universe and a cosmological constant, Astron. J. 116, 1009 (1998).

[43] L. Anderson et al., The clustering of galaxies in the SDSS-III baryon oscillation spectroscopic survey: Baryon 
acoustic oscillations in the data release 9 spectroscopic galaxy sample, Mon. Not. R. Astron. Soc. 427, 3435 (2012).

[44] P. de Bernardis et al., A flat universe from high-resolution maps of the cosmic microwave background radiation, Nature (London) 404, 955 (2000).

[45] T. F. Slater, J. P. Adams, G. Brissenden, and D. Duncan, What topics are taught in introductory astronomy courses?, Phys. Teach. 39, 52 (2001).

[46] J. W. Creswell and V.L. Plano Clark, Designing and Conducting Mixed Methods Research, 2nd ed. (SAGE Publications, Thousand Oaks, CA, 2011).

[47] R. J. Beichner, An introduction to physics education research, in Getting Started in PER: Reviews in PER, edited by C. Henderson and K. A. Harper (American Association of Physics Teachers, College Park, MD, 2009).
[48] J. Kregenow, M. Rogers, and M. Constas, Multidimensional education research: Managing multiple data streams, Astron. Educ. Rev. 9, 010104 (2010).

[49] S. Stemler, An overview of content analysis, Prac. Assess. Res. Eval. 7 (2001).

[50] J. W. Creswell, Qualitative Inquiry and Research Design: Choosing among Five Traditions, 2nd ed. (Sage Publications, Thousand Oaks, CA, 2007).

[51] K. L. Gwet, Handbook of Inter-Rater Reliability, 3rd ed. (Advanced Analytics, Gaithersburg, MD, 2012).

[52] E. M. Nussbaum, Categorical and Nonparametric Data Analysis: Choosing the Best Statistical Technique (Routledge, New York, NY, 2015).

[53] R. Driver and J. Easley, Pupils and paradigms: A review of literature related to concept development in adolescent science students, Stud. Sci. Educ. 5, 61 (1978). 